\title{
HSV-1 ICP27 induces apoptosis by promoting Bax translocation to mitochondria through interacting with
} $14-3-3 \theta$

\author{
Ji Ae Kim", Jin Chul Kim ${ }^{\text {+,\# }}$, Jung Sun Min, Inho Kang, Jeongho Oh E Jeong Keun Ahn ${ }^{*}$ \\ Department of Microbiology \& Molecular Biology, College of Biological Science and Biotechnology, Chungnam National University, \\ Daejeon 34134, Korea
}

\begin{abstract}
The subcellular localization of Bax plays a crucial role during apoptosis. In response to apoptotic stimuli, Bax translocates from the cytoplasm to the mitochondria, where it promotes the release of cytochrome $\mathrm{c}$ to the cytoplasm. In cells infected with HSV-1, apoptosis is triggered or blocked by diverse mechanisms. In this study, we demonstrate how HSV-1 ICP27 induces apoptosis and modulates mitochondrial membrane potential in HEK 293T cells. We found that ICP27 interacts with 14-3-3 $\theta$ which sequesters Bax to the cytoplasm. In addition, ICP27 promotes the translocation of Bax to the mitochondria by inhibiting the interaction between 14-3-30 and Bax. Our findings may provide a novel apoptotic regulatory pathway induced by ICP27 during HSV-1 infection. [BMB Reports 2017; 50(5): 257-262]
\end{abstract}

\section{INTRODUCTION}

In mammals, apoptosis plays critical roles during normal development, maintenance of homeostasis, aging, and defense (1). Apoptosis is triggered and regulated by diverse stimuli and conditions. The extrinsic signaling pathway of apoptosis is induced by ligation of transmembrane death receptors and death ligands $(2,3)$, whereas the intrinsic signaling pathway is triggered by non-receptor-mediated stimuli such as oxidative stress, DNA damage, and ER stress. All these stimuli disrupt the mitochondrial membrane integrity, and consequently release cytochrome $\mathrm{c}$ into the cytoplasm (4). Bcl-2 family

*Corresponding author. Tel: +82-42-821-6418; Fax: +82-42-8227367; E-mail: jkahn@cnu.ac.kr

${ }^{\dagger}$ Current address: Division of Biological Sciences, University of California, San Diego, La Jolla, CA, USA

${ }^{\#}$ These authors contributed equally to this work.

https://doi.org/10.5483/BMBRep.2017.50.5.023

Received 9 February 2017, Revised 10 February 2017, Accepted 3 March 2017

Keywords: Apoptosis, Bax, ICP27, 14-3-30 proteins regulate the apoptotic mitochondrial events. Bcl-2 family members are comprised of anti-apoptotic members (Bcl-2, Bcl-w, and $\mathrm{BCl}-\mathrm{x}_{\mathrm{L}}$ ), multidomain pro-apoptotic members (Bax and Bak), and $\mathrm{BH} 3$ domain-only pro-apoptotic members (Bad, Bid, Bim, Bik, Noxa, and PUMA) (5, 6). A pro-apoptotic $\mathrm{Bcl}-2$ family member, Bax, has an essential role in inducing apoptosis in response to stress stimuli (7-9). Bax is mainly localized in the cytoplasm, but apoptotic stimulation promotes its redistribution into the mitochondrial membrane. After translocation to the mitochondria, Bax forms homo-oligomeric protein complexes that alter the mitochondrial membrane permeability (10). In healthy cells, Bax is sequestered to the cytoplasm by the 14-3-3 proteins (11).

Herpes simplex virus type 1 (HSV-1) is a neurotropic virus, replicating lytically during acute infection and establishing latency in peripheral neurons. During lytic infection, the $\mathrm{HSV}-1$ genes are expressed in a tightly regulated temporal cascade, involving the sequential expression of immediate early (IE), early (E), and late (L). IE proteins include ICPO, ICP4, ICP22, ICP27, and ICP47, and are implicated in regulating the viral early gene expression and host cellular proteins $(12,13)$. ICP27 is a multifunctional protein essential for viral replication, and plays pivotal roles in the switch from early to late gene expression. ICP27 is also implicated in the nuclear export of viral mRNA, and represses the host protein synthesis by inhibiting cellular mRNA splicing (14). In addition, ICP27 is involved in cell cycle control and the activation of signal pathways (15).

This study confirms that ICP27 induces apoptosis in 293T cells. Furthermore, we demonstrate that ICP27 promotes the mitochondrial translocation of Bax, via inhibition of the cytoplasmic sequestration of Bax by 14-3-30. These findings imply that ICP27 plays a critical role in the apoptotic induction of HSV-1 infected cells.

\section{RESULTS}

ICP27 induces apoptosis

To date, the effect of ICP27 on apoptosis in HSV-1 infected cells remains unclear. In order to clarify whether ICP27

ISSN: 1976-670X (electronic edition)

Copyright (C) 2017 by the The Korean Society for Biochemistry and Molecular Biology

(c) This is an open-access article distributed under the terms of the Creative Commons Attribution Non-Commercial License (http://creativecommons.org/licenses/by-nc/4.0) which permits unrestricted non-commercial use, distribution, and reproduction in any medium, provided the original work is properly cited. 
induces apoptosis, we first expressed ICP27 in HEK 293T cells and monitored apoptosis by using the Hoechst 33258 staining assay, to visualize the apoptotic nuclear morphological alterations. Evidently, the chromatin condensation increased in ICP27 expressing cells (Fig. 1A). Next, we performed flow cytometric analysis with Annexin V-FITC/PI dual staining to quantify the extent of apoptosis. As shown in Fig. 1B, the percentage of the apoptotic cells in the upper right quadrant, which were positive for both Annexin V-FITC and PI, increased in the presence of ICP27. By Western blotting, we further analyzed the effect of ICP27 on caspase 3 in 293T cells. ICP27 elevates the process of procaspase 3 to active caspase 3, an executor in apoptosis. Moreover, ICP27 increased the cleavage of poly (ADP-ribose) polymerase (PARP), which is a major substrate of caspase 3 (Fig. 1C). Collectively, these data indicate that ICP27 induces apoptosis in 293T cells.

\section{ICP27-induced apoptosis is associated with mitochondrial dysfunction}

The mitochondrial membrane potential (MMP) was evaluated in order to determine how ICP27 induces apoptosis. Dysfunction of the MMP is an important feature of mitochondrial damage. Flow cytometric assay with Rhodamine 123 staining showed that ICP27 increased the percentage of cells with low MMP (Fig. 2A). This result provided the evidence that ICP27induced apoptosis in 293T cells is associated with mitochondrial dysfunction. The MMP is tightly regulated by the Bcl-2 family
A
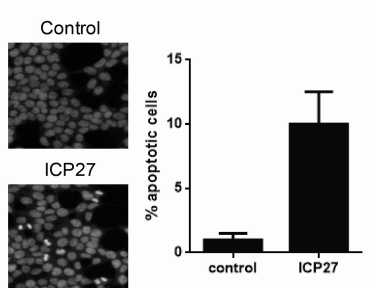
GST-ICP27 -

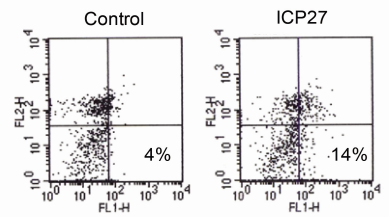

Fig. 1. HSV-1 ICP27 induces apoptosis. (A) Chromatin condensation of 293T cells induced by ICP27. Cells were transfected with ICP27 plasmid and stained with Hoechst 33258. Arrows indicate cells showing chromatin condensation. The graph represents three independent experiments, in which 200 cells were counted and scored for chromatin condensation. (B) Flow cytometry analysis of ICP27-induced 293T cell apoptosis. At $48 \mathrm{~h}$ after transfection, cells were stained with Annexin V-FITC/PI, and analyzed by flow cytometry. (C) Effect of ICP27 on the expressions of the apoptotic indicators caspase 3 and PARP, in 293T cells. At $48 \mathrm{~h}$ after transfection with ICP27 plasmid, cell extracts were prepared and analyzed by Western blotting with specific antibodies. proteins. Therefore, we next investigated the effect of ICP27 on the localization of Bax, a representative apoptotic Bcl-2 family protein, which, when activated, translocates to the mitochondria from the cytoplasm. The localization of Bax was assessed by staining the cells expressing GFP-Bax and ICP27 with Mitotracker, a mitochondrial-specific red fluorescent dye. Microscopic data revealed that in the presence of ICP27, GFP-Bax was localized on mitochondria (Fig. 2B). The Bax translocation was also confirmed by a subcellular fractionation assay. As expected, ICP27 increased the Bax translocation from the cytoplasm to the mitochondria (Fig. 2C). Further evaluation confirmed that ICP27 induces cytochrome c release from the mitochondria to the cytoplasm (Fig. 2D).

\section{ICP27 interferes with the interaction between Bax and 14-3-30}

To delineate the mechanism underlying ICP27-induced translocation of Bax, we speculated that ICP27 might affect the sequestration of Bax by 14-3-3, because 14-3-3 is not only an

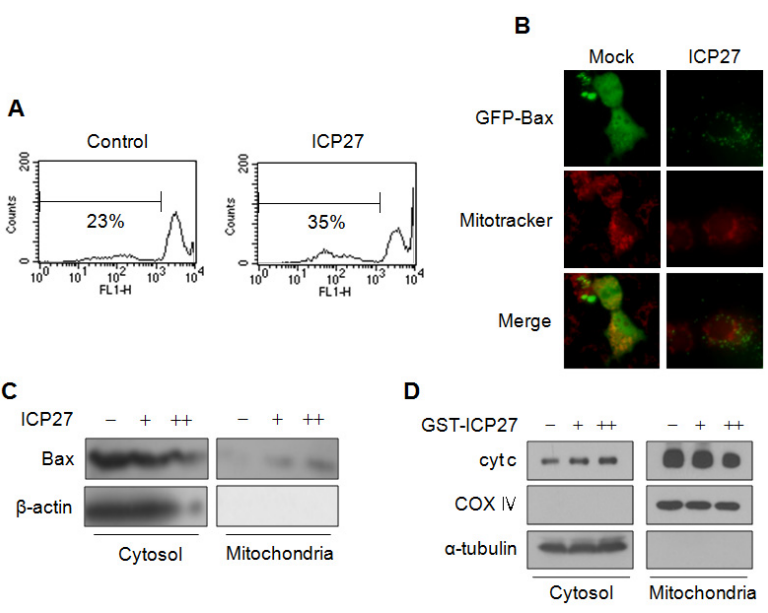

Fig. 2. ICP27 induces translocation of Bax and release of cytochrome $\mathrm{C}$ from the mitochondria. (A) Effect of ICP27 on the mitochondrial membrane potential in 293T cells. Cells were transfected with ICP27 expressing plasmid for $48 \mathrm{~h}$, and stained with Rhodamine 123 for $30 \mathrm{~min}$. The fluorescence intensity was measured by flow cytometry. (B) Subcellular localization of Bax by ICP27. Cells were transfected with plasmids expressing GFP-Bax and Flag-ICP27. At $48 \mathrm{~h}$ after transfection, cells were stained with Mitotracker for 30 min. Subcellular distribution of GFP-Bax and mitochondria (red) were determined by immunofluorescence microscopy. (C) Subcellular distribution of Bax by ICP27 in 293T cells. At $48 \mathrm{~h}$ after transfection with ICP27 plasmid, cells were fractionated into cytoplasmic and mitochondrial fractions. Fractionated proteins were immunoblotted with anti-Bax antibody. $\beta$-actin was used as a cytosolic marker. (D) Cytochrome c release from the mitochondria by ICP27. At $48 \mathrm{~h}$ after transfection with ICP27 plasmid, cells were fractionated into cytoplasmic and mitochondrial fractions. Fractionated proteins were immunoblotted with anti-cytochrome $\mathrm{C}$ antibody. $\alpha$-tubulin was used as a cytosolic marker and COX IV was used as a mitochondrial marker. 
important regulator for cytosolic sequestration of Bax but is also one of cellular proteins screened in the yeast two-hybrid assay using ICP27 as a bait (data not shown) (11). First, we checked the physical interaction between ICP27 and 14-3-3 by the pull-down assay. Among the seven 14-3-3 isoforms, 14-3-3 $\theta$ was selected for evaluation, since it has been reported that 14-3-30 is involved in subcellular localization of Bax (11). As seen in Fig. 3A, ICP27 interacts directly with 14-3-30. Further examination revealed that the interaction between 14-3-30 and Bax was inhibited by ICP27 (Fig. 3B). The mechanism how ICP27 inhibits the interaction between 14-3-30 and Bax was investigated by examining whether ICP27 regulates the acetylation of 14-3-30. The interaction between 14-3-3 and its target protein is regulated by the phosphorylation and acetylation state of 14-3-3 (16-18). As shown in Fig. 3C, ICP27 decreases the acetylation level of 14-3-30, which is regulated by SIRT2, a member of the class III HDAC family (16). Therefore, we speculate that ICP27 elevates

\section{A}

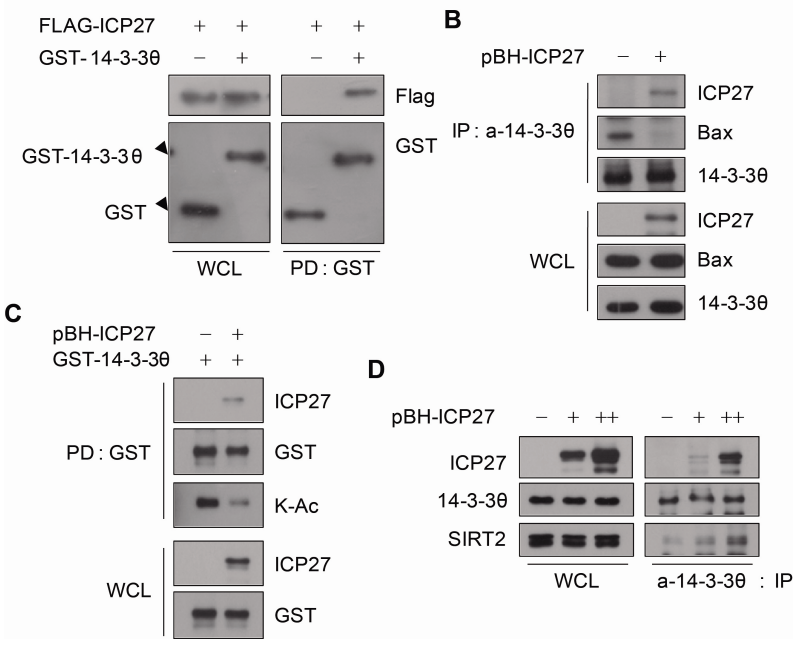

Fig. 3. ICP27 inhibits the association between Bax and 14-3-30. (A) Interaction between 14-3-30 and ICP27 in 293T cells. Cells were transfected with plasmids expressing Flag-ICP27 and GST14-3-30. The cell extracts were pulled-down with GlutathioneSepharose 4B beads, and the protein complexes were analyzed by immunoblotting using anti-Flag and anti-GST antibodies. (B) Effect of ICP27 on the interaction between Bax and 14-3-30. Extracts of 293T cells transfected with ICP27 plasmid were immunoprecipitated with anti-14-3-30 antibody. Immunocomplexes were analyzed by immunoblotting, using specific antibodies. (C) Effect of ICP27 on the level of 14-3-30 acetylation. Extracts of 293T cells expressing Flag-ICP27 and GST-14-3-30 were prepared. GST14-3-30 was pulled-down with Glutathione-Sepharose $4 \mathrm{~B}$ beads, and the level of 14-3-30 acetylation was analyzed by immunoblotting with acetyl-lysine antibody. (D) Effect of ICP27 on the association between 14-3-30 and SIRT2. 293T cells were transfected with ICP27 plasmid. Cell lysates were immunoprecipitated with anti-14-3-30 antibody, and immunocomplexes were analyzed by immunoblotting with SIRT2 antibody. the interaction between 14-3-30 and SIRT2. In order to test this hypothesis, 293T cells expressing ICP27 were immunoprecipitated with 14-3-30 antibody and immunoblotted with the SIRT2 antibody. The result revealed that SIRT2 is coimmunoprecipitated with 14-3-30, and ICP27 increased the binding between 14-3-3 and SIRT2 (Fig. 3D). Taken together, these data suggest that ICP27 inhibits the interaction between Bax and $14-3-3 \theta$ by enhancing the 14-3-30 deacetylation through SIRT2.

\section{In HSV-1 infection, ICP27 inhibits the interaction between Bax and 14-3-3}

We next investigated whether ICP27 affects Bax translocation during the early period of HSV-1 infection. 293T cells were infected with HSV-1 at MOI 10. At 6 h post infection, 293T cells were harvested and immunoprecipitated with 14-3-30 antibody. In HSV-1 infected cells, there was decreased interaction between Bax and 14-3-30, compared to the mock-infected cells (Fig. 4A). To further clarify the interaction between Bax and 14-3-3 affected by ICP27, 293T cells were infected with the HSV- 1 wt virus and $\Delta 27$ virus. In HSV- 1 wt-infected cells, the interaction between Bax and 14-3-3 $\theta$ was significantly decreased when compared with mock and $\Delta 27$ infected cells (Fig. 4B). Thus, in HSV-1 infection, ICP27 inhibits the interaction between Bax and 14-3-30, by interacting with 14-3-30.

\section{DISCUSSION}

Previous reports indicate that ICP27 is associated with apoptosis in HSV-1 infected cells $(19,20)$. However, it remains unclear whether ICP27 induces or inhibits apoptosis. Aubert et al. showed that ICP27 is required for the prevention of apoptosis using ICP27 deletion mutant virus $(21,22)$. Conversely, other studies showed that IE gene expression is required for apoptosis induction, and ICP27 induces apoptosis through activating the p38 mitogen-activated protein kinase (MAPK) signaling $(19,23)$. ICP27 regulates viral gene expression, and it is possible that other viral proteins regulated by ICP27 may also affect apoptosis. Hence, it would be inappropriate to investigate the role of ICP27 in regulating apoptosis using

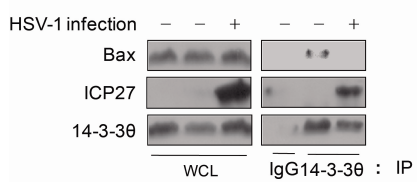

B

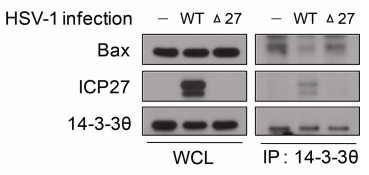

Fig. 4. ICP27 inhibits the interaction between Bax and 14-3-30 at the early stage of HSV-1 infection. Interaction between Bax and $14-3-3 \theta$ in virus infected cells. 293T cells were infected with HSV-1 virus (A) and HSV-1 $\Delta 27$ virus (B) at MOl of 10 . At $6 \mathrm{~h}$ post infection, cells were harvested and immunoprecipitated with anti-14-3-3 $\theta$ antibody. Immunocomplexes were analyzed by immunoblotting with specific antibodies. 
ICP27 deletion virus exclusively, and it is more suitable to assess the role of ICP27 in apoptosis by using the protein itself.

Apoptosis is mediated by diverse stimuli, which induce the disruption of the mitochondrial membrane permeability. The consequent release of cytochrome $\mathrm{c}$ from the mitochondria to the cytoplasm in response to apoptotic stimulation is a critical event in apoptosis. The mitochondrial membrane integrity is tightly regulated by $\mathrm{Bcl}-2$ family proteins. Particularly, Bax oligomerizes and translocates to the mitochondrial outer membrane, where it forms supramolecular openings under apoptotic stimuli $(24,25)$. Many studies have shown that diverse viral proteins induce apoptosis by regulating the $\mathrm{BCl}-2$ family proteins. For example, hepatitis $B$ virus $X$ protein induces apoptosis via enhancement of the Bax translocation (26). Our data support the reports that viral protein induces apoptosis through modulation of the Bcl-2 family protein. We showed that ICP27 induces apoptosis in 293T cells through mitochondrial dysfunction. We also found that ICP27 enhances the translocation of Bax to the mitochondria.

The subcellular localization of Bax is regulated by the 14-3-3 proteins. The 14-3-3 proteins interact with Bax, sequestering it to the cytoplasm. Recent studies show that posttranslational modification of 14-3-3 proteins is important for its binding activity with target proteins (27). Phosphorylation of 14-3-3 proteins leads to self-dimerization, and affects the interaction with target proteins (28). SDK1 and JNK phosphorylate the 14-3-3 proteins, leading to the dissociation of Bax from the 14-3-3 proteins $(29,30)$. Lysine acetylation of 14-3-3 proteins also affects the interaction with target proteins, but the effect remains ambiguous. We showed that ICP27 decreases the acetylation level of $14-3-3 \theta$ by enhancing the interaction between SIRT2 and 14-3-30, and subsequently inhibits the interaction between 14-3-30 and Bax.

These results suggest new molecular mechanisms of apoptosis modulated by the HSV-1 ICP27.

\section{MATERIALS AND METHODS}

\section{Cells and reagents}

HEK 293T, Vero, and Vero 3-3 (HSV-1 ICP27 expressing Vero) cells were maintained in Dulbecco's modified Eagle's medium (DMEM) supplemented with $10 \%$ fetal bovine serum (FBS), at $37^{\circ} \mathrm{C}$ in $5 \% \mathrm{CO}_{2}$. Cells were transfected using PEI (SigmaAldrich), according to the manufacturer's instruction. Trichostatin A (TSA) was purchased from Sigma-Aldrich.

\footnotetext{
Viruses and virus infection

HSV-1 wild-type (wt) strain KOS and ICP27 deletion mutant $(\Delta 27)$ virus $(31)$ were propagated and titrated on Vero cells and 3-3 cells, respectively. For virus infection, cell monolayers were infected with either KOS or mutant virus, at a multiplicity of infection (MOI) of 10 plaque forming units (PFU)/cell.
}

\section{Plasmids}

ICP27 plasmids have been described previously (32). $\mathrm{pBH}$ ICP27 was provided by Dr. Stephen A. Rice (33). Plasmids expressing pEGFP-SUMO-1 and HA-UBC9 were provided by Dr. Hsiu-Ming Shih (34) and Dr. H. Ariga (35), respectively. pEGFP-Bax was provided by Dr. Robert W. Nickells (36).

\section{Detection of apoptosis (Hoechst 33258 staining)}

At $48 \mathrm{~h}$ after transfection, cells were washed with phosphatebuffered saline (PBS), fixed with $4 \%$ paraformaldehyde for 15 min, and then permeabilized with $0.05 \%$ Triton X-100. The fixed cells were washed with PBS and stained with $1 \mathrm{mg} / \mathrm{ml}$ Hoechst 33258 (Sigma-Aldrich) for $10 \mathrm{~min}$ (37). Apoptosis of the cells was determined by chromatin condensation, under a fluorescent microscope (Olympus).

\section{Annexin- $\mathrm{V}$ apoptosis assay}

At $48 \mathrm{~h}$ after transfection, the level of apoptosis was detected using the Annexin V-FITC Apoptosis detection kit (BD Pharmingen), according to the manufacturer's manual (38). Flow-cytometric analysis was performed using a Becton Dickinson FACS Calibur flow cytometer and Cell Quest software (Becton Dickinson).

\section{Detection of the MMP}

The MMP was measured by using rhodamine-123 (Rh123) (39). Cells were harvested by trypsinization, and re-suspended in medium at a density of $5 \times 10^{4}$ cells $/ \mathrm{ml}$. The cells were then incubated with $5 \mu \mathrm{M}$ Rh123 at room temperature for 20 min, washed twice with PBS, re-suspended in tissue culture medium, and analyzed by a Becton Dickinson FACS Calibur flow cytometer.

\section{Subcellular fractionation assay}

Briefly, cells were suspended in fractionation buffer $(250 \mathrm{mM}$ sucrose, $20 \mathrm{mM}$ HEPES pH 7.4, $10 \mathrm{mM} \mathrm{KCl}, 1.5 \mathrm{mM} \mathrm{MgCl}$, 1 mM EDTA, 1 mM EGTA, 1 mM DTT, 1 mM PMSF, 1 mM NaF, $1 \mathrm{mM} \mathrm{Na} \mathrm{VO}_{4}, 1 \mu \mathrm{g} / \mathrm{ml}$ aprotinin, $1 \mu \mathrm{g} / \mathrm{ml}$ leupeptin, and 1 $\mu \mathrm{g} / \mathrm{ml}$ pepstatin). The cell lysate was passed 10 times through a 25 ga needle using a $1 \mathrm{ml}$ syringe, and stored on ice for 20 $\mathrm{min}$. The lysates were centrifuged at $3000 \mathrm{rpm}$ at $4^{\circ} \mathrm{C}$ for 5 $\mathrm{min}$, and the supernatants were collected as cytosolic fractions. The nuclear pellets were re-suspended in the nuclear buffer (RIPA buffer with $10 \%$ glycerol and $0.1 \%$ SDS), and sonicated in three $10 \mathrm{sec}$ bursts at $40 \%$ intensity, with $30 \mathrm{sec}$ pauses on ice.

\section{GST pull-down assay}

Transiently transfected 293T cells were lysed in modified RIPA buffer $(50 \mathrm{mM}$ Tris- $\mathrm{HCl} \mathrm{pH} 7.4,1 \% \mathrm{NP}-40,0.25 \% \mathrm{Na}-$ deoxycholate, $150 \mathrm{mM} \mathrm{NaCl}, 1 \mathrm{mM}$ EDTA, $1 \mathrm{mM}$ PMSF, 1 $\mathrm{mM} \mathrm{NaF}, 1 \mathrm{mM} \mathrm{Na} \mathrm{VO}_{4}, 1 \mu \mathrm{g} / \mathrm{ml}$ aprotinin, $1 \mu \mathrm{g} / \mathrm{ml}$ leupeptin, and $1 \mu \mathrm{g} / \mathrm{ml}$ pepstatin) at $4^{\circ} \mathrm{C}$ for $30 \mathrm{~min}$. The cell lysates were incubated overnight at $4^{\circ} \mathrm{C}$ with Glutathione-Sepharose $4 \mathrm{~B}$ 
beads (Incospharm). The bead complexes were washed three times with modified RIPA buffer, and the bound proteins were analyzed by Western blotting.

\section{Immunoprecipitation and Western blot analysis}

Cell lysates were prepared in modified RIPA buffer and incubated overnight at $4^{\circ} \mathrm{C}$, with specific antibodies. The immunocomplexes were isolated using protein-A Sepharose (Incospharm), resolved by SDS-PAGE, and transferred to nitrocellulose membrane. Immunoblotting was performed with various antibodies. Anti-ICP27, anti-Bax, anti-14-3-30, anticytochrome $\mathrm{C}$, anti-COX IV, anti- $\alpha$-tubulin, anti- $\beta$-actin, antiacetylated lysine, anti-SIRT2, goat anti-rabbit IgG-HRP, rabbit anti-goat IgG-HRP, and goat anti-mouse IgG-HRP antibodies were purchased from Santa Cruz. Anti-Flag and anti-GST antibodies were purchased from Sigma-Aldrich.

\section{Immunofluorescence assay}

Vero cells were plated on sterile glass coverslips and transfected with GFP-Bax and GST-ICP27. At $48 \mathrm{~h}$ after transfection, cells were incubated with $20 \mathrm{ng} / \mathrm{ml}$ of a mitochondrial-specific dye (Mitotracker red CMXRos; Cambrex BioScience) for 30 min. Cells were fixed with $4 \%$ paraformaldehyde, at room temperature for $10 \mathrm{~min}$, and permeabilized for $5 \mathrm{~min}$ with PBS containing $0.2 \%$ Triton X-100. Fluorescence microscopy was performed using an Olympus microscope.

\section{ACKNOWLEDGEMENTS}

This work was supported by the research fund of Chungnam National University.

\section{CONFLICTS OF INTEREST}

The authors have no conflicting financial interests.

\section{REFERENCES}

1. Elmore S (2007) Apoptosis: a review of programmed cell death. Toxicol Pathol 35, 495-516

2. Ashkenazi A and Dixit VM (1998) Death receptors: signaling and modulation. Science 281, 1305-1308

3. Schmitz I, Kirchhoff S and Krammer PH (2000) Regulation of death receptor-mediated apoptosis pathways. Int J Biochem Cell Biol 32, 1123-1136

4. Wang $X$ (2001) The expanding role of mitochondria in apoptosis. Genes Dev 15, 2922-2933

5. Cory S and Adams JM (2002) The Bcl2 family: regulators of the cellular life-or-death switch. Nat Rev Cancer 2, 647-656

6. Martinou JC and Green DR (2001) Breaking the mitochondrial barrier. Nat Rev Mol Cell Biol 2, 63-67

7. Knudson CM, Tung KS, Tourtellotte WG, Brown GA and Korsmeyer SJ (1995) Bax-deficient mice with lymphoid hyperplasia and male germ cell death. Science 270, 96-99

8. Wei MC, Zong WX, Cheng EH et al (2001) Proapoptotic
BAX and BAK: a requisite gateway to mitochondrial dysfunction and death. Science 292, 727-730

9. Tsuruta F, Sunayama J, Mori Y et al (2004) JNK promotes Bax translocation to mitochondria through phosphorylation of 14-3-3 proteins. EMBO J 23, 1889-1899

10. Kuwana T, Mackey MR, Perkins G et al (2002) Bid, Bax and lipids cooperate to form supramolecular openings in the outer mitochondrial membrane. Cell 111, 331-342

11. Nomura M, Shimizu S, Sugiyama T et al (2003) 14-3-3 Interacts directly with and negatively regulates proapoptotic Bax. J Biol Chem 278, 2058-2065

12. Honess RW and Roizman B (1974) Regulation of herpesvirus macromolecular synthesis. I. Cascade regulation of the synthesis of three groups of viral proteins. J Virol 14, 8-19

13. Smiley JR (2004) Herpes simplex virus virion host shutoff protein: immune evasion mediated by a viral RNase? J Virol 78, 1063-1068

14. Chen IH, Li L, Silva L and Sandri-Goldin RM (2005) ICP27 recruits Aly/REF but not TAP/NXF1 to herpes simplex virus type 1 transcription sites although TAP/NXF1 is required for ICP27 export. J Virol 79, 3949-3961

15. Song BW, Yeh KC, Liu J and Knipe DM (2001) Herpes simplex virus gene products required for viral inhibition of expression of G1-phase functions. Virology 290, 320-328

16. Lynn EG, McLeod CJ, Gordon JP, Bao JJ and Sack MN (2008) SIRT2 is a negative regulator of anoxia-reoxygenation tolerance via regulation of 14-3-3 zeta and BAD in $\mathrm{H} 9 \mathrm{c} 2$ cells. FEBS Lett 582, 2857-2862

17. Dougherty MK and Morrison DK (2004) Unlocking the code of 14-3-3. J Cell Sci 117, 1875-1884

18. Tzivion G and Avruch J (2002) 14-3-3 proteins: Active cofactors in cellular regulation by serine/threonine phosphorylation. J Biol Chem 277, 3061-3064

19. Sanfilippo CM, Chirimuuta FN and Blaho JA (2004) Herpes simplex virus type 1 immediate-early gene expression is required for the induction of apoptosis in human epithelial HEp-2 cells. J Virol 78, 224-239

20. Kim JC, Choi SH, Kim JK et al (2008) Herpes simplex virus type 1 ICP27 induces apoptotic cell death by increasing intracellular reactive oxygen species. Mol Biol 42, 413-420

21. Aubert M and Blaho JA (1999) The herpes simplex virus type 1 regulatory protein ICP27 is required for the prevention of apoptosis in infected human cells. J Virol 73, 2803-2813

22. Aubert M, Rice SA and Blaho JA (2001) Accumulation of herpes simplex virus type 1 early and leaky-late proteins correlates with apoptosis prevention in infected human HEp-2 cells. J Virol 75, 1013-1030

23. Gillis PA, Okagaki LH and Rice SA (2009) Herpes simplex virus type 1 ICP27 induces p38 mitogen-activated protein kinase signaling and apoptosis in HeLa cells. J Virol 83, 1767-1777

24. Wolter KG, Hsu YT, Smith CL, Nechushtan A, Xi XG and Youle RJ (1997) Movement of Bax from the cytosol to mitochondria during apoptosis. J Cell Biol 139, 12811292

25. Kuwana T, Mackey MR, Perkins G et al (2002) Bid, Bax, and lipids cooperate to form supramolecular openings in 
the outer mitochondrial membrane. Cell 111, 331-342

26. Kim HJ, Kim SY, Kim J et al (2008) Hepatitis B virus X protein induces apoptosis by enhancing translocation of Bax to mitochondria. IUBMB Life 60, 473-480

27. Aitken A (2011) Post-translational modification of 14-3-3 isoforms and regulation of cellular function. Semin Cell Dev Biol 22, 673-680

28. Aitken A (2002) Functional specificity in 14-3-3 isoform interactions through dimer formation and phosphorylation. Chromosome location of mammalian isoforms and variants. Plant Mol Biol 50, 993-1010

29. Kanno T and Nishizaki T (2011) Sphingosine induces apoptosis in hippocampal neurons and astrocytes by activating caspase-3/-9 via a mitochondrial pathway linked to SDK/14-3-3 protein/Bax/cytochrome c. J Cell Physiol 226, 2329-2337

30. Tsuruta F, Sunayama J, Mori Y et al (2004) JNK promotes Bax translocation to mitochondria through phosphorylation of 14-3-3 proteins. EMBO J 23, 1889-1899

31. McMahan L and Schaffer PA (1990) The repressing and enhancing functions of the herpes simplex virus regulatory protein ICP27 map to C-terminal regions and are required to modulate viral gene expression very early in infection. J Virol 64, 3471-3485

32. Kim JC, Lee SY, Kim SY et al (2008) HSV-1 ICP27 suppresses NF-kappaB activity by stabilizing IkappaBalpha. FEBS Lett 582, 2371-2376

33. Rice SA and Knipe DM (1988) Gene-Specific Transactivation by Herpes-Simplex Virus Type-1 Alpha-Protein-Icp27.
J Virol 62, 3814-3823

34. Kuo HY, Chang CC, Jeng JC et al (2005) SUMO modification negatively modulates the transcriptional activity of CREB-binding protein via the recruitment of Daxx. Proc Natl Acad Sci U S A 102, 16973-16978

35. Shinbo Y, Niki T, Taira T et al (2006) Proper SUMO-1 conjugation is essential to DJ-1 to exert its full activities. Cell Death Differ 13, 96-108

36. Semaan SJ and Nickells RW (2010) The apoptotic response in HCT116BAX-- cancer cells becomes rapidly saturated with increasing expression of a GFP-BAX fusion protein. BMC Cancer 10, 554

37. Abreu-Martin MT, Chari A, Palladino AA, Craft NA and Sawyers CL (1999) Mitogen-activated protein kinase kinase kinase 1 activates androgen receptor-dependent transcription and apoptosis in prostate cancer. Mol Cell Biol 19, 5143-5154

38. Miao L, Yi P, Wang Y and Wu M (2003) Etoposide upregulates Bax-enhancing tumour necrosis factor-related apoptosis inducing ligand-mediated apoptosis in the human hepatocellular carcinoma cell line QGY-7703. Eur J Biochem 270, 2721-2731

39. Li L, Lorenzo PS, Bogi K, Blumberg PM and Yuspa SH (1999) Protein kinase Cdelta targets mitochondria, alters mitochondrial membrane potential, and induces apoptosis in normal and neoplastic keratinocytes when overexpressed by an adenoviral vector. Mol Cell Biol 19, 8547-8558 